level ranges as other hospital equipment, and thus the machine should not pose an increased risk of exposure to electromagnetic fields. The system meets all codes, including stringent standards for radio-frequency emissions.

\section{SUMMAR Y}

The process of sterilization with hydrogen peroxide plasma is very different than with steam or ETO sterilization. Consequently, there is room for errors in processing, in areas such as packaging, especially when the system is first used. Personnel must be trained thoroughly in the process before using this system. I would recommend that this system only be used in central medical supply, where employees are experts in processing and the process is controlled.

Hydrogen peroxide plasma technology may be a breakthrough in the area of sterilization. Its applications may yet prove important to the healthcare industry, as the pressure to phase out potentially toxic sterilants such as ethylene oxide increases. Approxi- mately 100 sterilization units have been sold in the United States since FDA approval. Before purchasing this product, it is recommended that the customers of these systems be contacted to discuss its practical application.

\section{REFERENCES}

1. Jacobs PT Plasma sterilization. Journal of Healthcare Materiel Management 1989;7(5):49.

2. Hospital Purchasing News, Inc. New sterilizer could hasten ethylene oxide exodus. Hospital Purchasing News. 38-Passim, May 15, 1994.

3. Mecke P. Hydrogen peroxide plasma-an interesting micmbicidal concept. Hygiene + Medizin1992;17:537-543.

4. Advanced Sterilization Products Inc. Sterrad 100 Sterilization System, Operator's Manual, Preliminary Vision. Irvine, CA: Advanced Sterilization Products, Inc. 1993.

5. Jacobs PT. Sterrad Sterilization System: A New Technology for Instrument Sterilization. Irvine, CA: Advanced Sterilization Products, Inc. 1994.

6. Caputo RA, Fisher J, Jarzynski V, Martens PA Validation testing of a gas plasma sterilization system. Medical Devise and Diagnostic Industry 1993;15:132-138.

7. Patterson P, ed. Sterilization alternatives: new techniques needed as CDCs are taken off market. OR Manager 1994;10(8).

\title{
Increased Risk of Bloodstream Infection with Needleless Device
}

\section{by Gina Pugliese, RN, MS Medical News Editor}

The CDC recently reported the results of an investigation of an increase in the number of bloodstream infections (BSIs) in patients receiving home intravenous therapy. The investigation determined that a needleless device used for administration of total parenteral nutrition with intralipid admixture (TPN/IL) through a central venous catheter was associated with an increased risk of BSIs when the injection caps were changed every 7 days.

The researchers compared patients receiving home therapy infusion who developed BSI (case patients) with randomly selected noninfected home care patients receiving intravenous therapy and conducted a cohort study of all home therapy patients receiving intravenous therapy via a central venous catheter (CVC).

The results indicated that cases were more likely than controls to have had therapy via a CVC (11 of 11 versus 14 of 32) or receive TPN/IL (9 of 11 versus 3 of 32 ). Among the home intravenous therapy patients with CVCs, risk factors for BSIs were receipt of TPN/IL or use of a needleless infusion system.

A procedural review indicated that two types of infusion access devices had been used. Initially, a protected-needle access system (Clicklock, ICU Medical, San Clemente, CA) was used that included an injection cap with a latex injection site and an access needle that was in a protective housing and snapped in place over the latex injection cap site. The caps were changed every 7 days, and the needle and its housing were changed daily. This system was replaced with a needleless infusion device (Interlink Access System, Baxter Health Care, Round Lake, IL) that included a pre-slit latex injection (cap) site with a blunt cannula housed in a threaded locking device that fit over the injection site. The cap with latex injection site was changed every 7 days, and the blunt cannula (used for entry into the injection site) was changed daily. Culture surveys indicated that luminal fluid from injection caps of the needleless devices was significantly more likely to be culture positive than fluid from protected-needle devices ( 5 of 23 versus 0 of 18 ).

The exact mechanism whereby TPN/IL and the needleless system resulted in an increased risk of BSI is not known. The researchers noted that a likely mechanism, suggested by the culture survey of luminal fluid of injection caps, was compromised sterility of the luminal contents of the pre-slit latex injection cap sites (compared with the injection sites used with protected needles). Reasons for such contamination included multiple manipulations of the cannula tip and injection site over the 7 days before injection cap change. It was hypothesized that in patients with CVCs, TPN/IL, and needleless devices, the nutrient-rich solutions remained in the injection cap and became contaminated during injection cap manipulations. During the 7 days before the injection caps were changed, the contaminating pathogens proliferated and then caused BSIs.

This study illustrates the need for defining infection control standards for needleless devices and conducting surveillance for adverse events after their introduction.

FROM: Danzig L, Short LJ, Collins $\mathrm{K}$, et al. Bloodstream infections associated with a needleless intravenous infusion system in patients receiving home infusion therapy. JAMA 273 (23) :18621864. 